\title{
Hierarchical DNA Memory based on Nested PCR
}

\author{
Satoshi Kashiwamura ${ }^{1}$, Masahito Yamamoto ${ }^{1}$, Atsushi Kameda ${ }^{2}$, \\ Toshikazu Shiba ${ }^{3}$, and Azuma Ohuchi ${ }^{3}$ \\ 1 Graduate School of Engineering, Hokkaido University \\ North 13, West 8, Kita-ku, Sapporo 060-8628, Japan \\ \{kashiwa, masahito\}@dna-comp.org \\ http://ses3. complex.eng.hokudai.ac.jp/index.html \\ 2 Japan Science and Technology Cooperation (JST) \\ Honmachi 4-1-8, Kawaguchi 332-0012, Japan \\ kameda@dna-comp.org \\ ${ }^{3}$ CREST, Japan Science and Technology Cooperation (JST) and \\ Graduate School of Engineering, Hokkaido University \\ North 13, West 8, Kita-ku, Sapporo 060-8628, Japan \\ \{shiba, ohuchi\}@dna-comp.org
}

\begin{abstract}
This paper presents a hierarchical DNA memory based on nested PCR. Each memory consists of address blocks and a data block. In order to access specific data, we specify the order of the address primers, and nested PCR are performed by using these primers. Our laboratory experiments are also presented to demonstrate the feasibility of the proposed memory.
\end{abstract}

\section{Introduction}

DNA is an excellent memory unit because it has the capability to store vast amount of data in a very small space. Focusing on this aspect of DNA, our study aims to propose and to construct DNA memory with high memory capacity at an extremely minute scale. We present an implementation of a hierarchical DNA memory based on nested PCR, which is called Nested Primer Molecular Memory (NPMM). In an NPMM, a data block sequence is concatenated with address block sequences. Address blocks consist of primers for the addressing of data. In order to access specific data, we specify the order of address primers, and nested PCR are performed by using these primers. In the NPMM systems, a high-level reaction specificity and a very secure memory system can be achieved.

In this paper, we focus on the addressing of the data but do not discuss the encoding of the data into DNA sequences or the decoding of the sequences. The structure and design strategy of NPMM is described, and our laboratory experiments are presented in order to demonstrate the feasibility of NPMM. 


\section{Related Works}

The most famous work on Molecular Memory may be the efforts of Head et al., who proposed memory with an aquatic feature. In their approach, any problem could be solved by handling aquatic memory appropriately. This is called as aqueous computing [1][2]. Their idea overcomes the overhead of the sequence design problem, which is a serious issue in conventional DNA computation. On this point, their memory is an excellent breakthrough, but it does not focus on achieving the high memory capacity and compactness of DNA. Actually, it may be difficult to enlarge their memory system experimentally.

On the other hand, Baum was the first to proposed to constructing memory by focusing on the high memory capacitance and compactness of DNA[3]. In his model, a massively parallel associative search in a vast database made of DNA can be realized by affinity separation methods. Rief et al. refined the previous memory idea by using computational and informational processing[4], and large DNA memory was constructed experimentally[5]. They decreased the reaction error in memory by a computational and information technique. However, when the memory grows larger, in other words, the number of DNA sequences to be designed increases, the reaction specificity of each DNA strand will get smaller. This implies that errors could easily occur. Therefore, it will also be necessary to make efforts to decrease the number of DNA strand to be designed.

Here, we propose a new DNA memory system based on PCR. Our system has new advantages that were not in the previous models, which are described in the next section. In this paper, we do not discuss the associative search in our model, but associative search will become possible by using a few devices.

\section{Nested Primer Molecular Memory}

In the case of conventional memory, data is encoded to $\{0,1\}$ bit sequence data and then stored in media. In the case of Nested Primer Molecular Memory (NPMM), data is encoded to $\{\mathrm{A}, \mathrm{T}, \mathrm{G}, \mathrm{C}\}$ base sequence data and then stored in a DNA strand. NPMM is a aqueous solution in which DNA (data) are mixed, and each DNA sequence in NPMM consists of the areas where stored data and the data address are expressed. If we specify the address of target data, we can get the target data from NPMM.

\subsection{Data Structure of NPMM}

NPMM consists of some DNA sequences in which data are stored. Each DNA sequence consists of three types of blocks: data block, some address blocks, and Re block.

- Data block is the site where data are encoded into a base $\{A, T, G, C\}$ sequence. 
- Address blocks are the sites where addresses are specified and consist of some blocks at the 5'end of the data block.

- Re block is the site where the reverse primer should be hybridized, and is the common sequence in all DNA sequences in NPMM.

In this paper, the number of address blocks is fixed to three (named A block, $\mathrm{B}$ block, $\mathrm{C}$ block) and the number of sequences in each address block is three (labeled $0,1,2$ ). The oligo (working as a forward primer) that should be hybridized to the sequence labeled $i(i \in\{0,1,2\})$ in $X$ block $(X \in\{\mathrm{A}, \mathrm{B}, \mathrm{C}\})$ is denoted by $X_{i}$. We define a reverse primer as $R e$, the data sequence following the $A_{i}, B_{j}, C_{k}$ annealing site as $d a t a_{i j k}$, and the overall DNA sequence as template $i j k$ (Fig. 1).

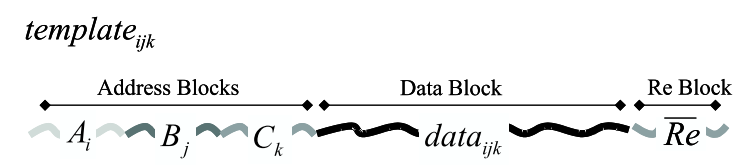

Fig. 1. Data structure of templates.

We define $P$ and $T$ as $P=\left\{A_{i}, B_{j}, C_{k}, R e \mid i, j, k \in\{0,1,2\}\right\}$ and $T=$ $\left\{\right.$ template $\left._{i j k} \mid i, j, k \in\{0,1,2\}\right\}$.

\subsection{Addressing the data}

The procedure to specify an address in NPMM is as follows.

1. Select a $p \in P$, and then perform PCR to NPMM with $p$ and Re.

2. Select another $p^{\prime} \in P$, then perform PCR to the diluted solution after the previous PCR.

3. Repeat step 2 for the appropriate number of times.

By following the above procedure, we can obtain one sequence coding the target data. A set of used $p$ and its order serve as the data address in NPMM, and we denote the address by $\left[p, p^{\prime}, p^{\prime \prime} \ldots\right]$. ( $p$ : forward primer used in first PCR, $p^{\prime}$ : primer in second PCR, $p^{\prime \prime}$ : and so on in this fashion.)

In order to deepen our understanding, we show an instance of extracting data $_{101}$ from NPMM in Fig. 2. We know the address of data $a_{101}$ is $\left[A_{1}, B_{0}, C_{1}\right]$. For the first PCR, $A_{1}$ and $R e$ are used in NPMM. Then, after PCR, the diluted solution consists of nine DNA containing an $A_{1}$ annealing site, because only those nine DNA are amplified. For the second PCR, $B_{0}$ and $R e$ are used in the diluted solution after the first PCR. Then, only three DNA containing a $B_{0}$ annealing site are amplified out of the nine DNA. Finally, $C_{1}$ and $R e$ are used in the diluted solution after the second PCR. As a result, there is only sequence coding data $_{101}$ in the solution; hence we can obtain the target data data $a_{101}$. 


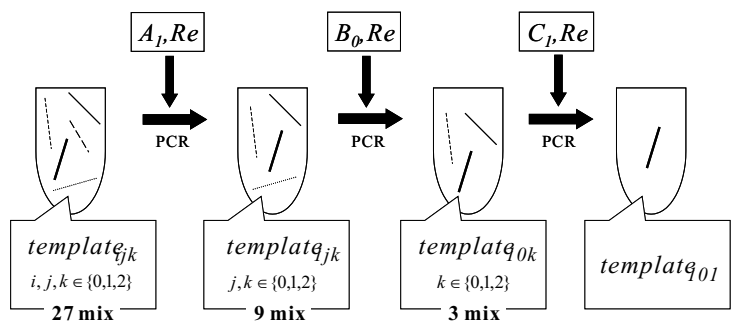

Fig. 2. One target data sequence is extracted from a 27-templates mix by addressing with three times PCR. (The shortening of the length of templates by PCR is omitted here.)

\subsection{Merits of NPMM}

\section{NPMM provides a high level of data security}

It is impossible to acquire a readout of the target data unless you can get primers. Accordingly, each primer works as a key, and each key is independent of other keys. In other words, the primers work as distributed keys. Furthermore, even if you could get all of the primers, the order of each primer to specify an address would be complicated in permutation, i.e. even in the case of the previous example shown in Fig.2, there are $5040\left(={ }_{10} P_{4}\right)$ ways of how to select primers.

Since we must get primers as keys and must know the order of each primer in order to acquire a readout of the specific data, a very secure memory system with some distributed keys would be realized.

\section{NPMM has a large capacity with a high reaction specificity}

The number of primers we should design is much less than that of data stored in NPMM. Because of the abundant use of PCR, a very high level of specificity of primers is absolutely essential for NPMM. Needless to say, an increase in the number of primers we have to design decreases the reaction specificity of the primers. NPMM realizes a large memory capacity in spite of a small number of primers. In fact, the capacity of NPMM grows exponentially with an increase in address blocks. The memory capacity of NPMM is determined as follows.

$$
M(b i t)=2 \times \operatorname{Data}(b p) \times \text { Primer }^{\text {Block }}
$$

$M$ : memory capacity of NPMM.

Data: length of the sequence in the data block.

Block: number of address blocks.

Primer: number of primers in each address block.

One base is equal to 2 bits because one base consists of 4 elements (A,T,G,C).

In addition, Using nested PCR raises the specificity of the final reaction products. [9] 


\section{Ease of extracting the target data from NPMM}

We can extract the target data from NPMM by using only PCR and Sequencing. PCR, as well as sequencing, is a very sophisticated, common-use, automated and easy tool. Therefore, anyone can extract the target data from NPMM easily, without special tools or experimental skill.

\subsection{Potential Applications}

NPMM can be used for media in which very secure data is stored. NPMM can also be used as media to store the data that is not usually referenced but shouldn't be erased (e.g. accumulated log files). Another application is media to store human genetic information. The advances in genetic information have posed privacy problems in recent years. At present, an individual's genetic information is read out and then converted to digital data. Genetic information is stored as this digital data in some media. Since genetic information is read out once, genetic information may leak out. On the other hand, the individual's genetic information could be saved in NPMM as a raw gene, without reading and translating individual genetic information. Accordingly, NPMM would protect the privacy of genetic information.

\section{NPMM design}

In order to investigate the feasibility of NPMM, we designed a small-sized NPMM. The evaluation function for the sequence design was originally formulated by consulting some references [6][7][8].

\subsection{Size of NPMM}

In this paper, we set the size of NPMM: $27\left(3^{3}\right)$ kinds of data can be stored in this NPMM, and the memory capacity is 135 bytes. The number of primers to be designed is $10(=3 \times 3+1)$. The other parameters are described as follows.

- length of data sequences: $20 \mathrm{bp}$

- length of primers $(p \in P):$ All $15 \mathrm{bp}$

- length of template $(t \in T): 80$ bp $(=15+15+15+20+15)$

\subsection{Design of sequences}

We designed data sequences and primer sequences with the following procedure.

1. Design 27 data sequences randomly.

2. Design 10 primer sequences so as to prevent mishybridization of each template and each primer by computer simulation as follows. 


\section{Strategy of primer design}

In this instance, we must design $\mathrm{P}$ because data sequences are designed randomly, and $T$ is created automatically when $P$ and data sequences are designed.

We took following the 3 evaluation items into consideration in designing $P$. Each evaluation value is calculated on each evaluation item.

1. GC_content

2. Hamming distance

3. 3'end_complementary

$P$ is designed so as to optimize the evaluation functions, which are calculated as linear sums of each evaluation value by Simulated Annealing Method.

\section{GC_content}

It is necessary to make the $\mathrm{Tm}$ (melting temperature) of each primer regularity to achieve a high specificity in the PCR reaction. Therefore, each primer should share the GC content. The evaluation value (GC_value) of GC_content on $P$ is calculated as follows.

$$
G C \_v a l u e=\max \left(\left(G C_{\text {define }}-G C_{p}\right)^{2}\right)+G C \_m a x \_n u m b e r /|P|,
$$

where $G C_{\text {define }}$ is the target value of the GC content of the primer and $G C_{p}$ is the GC content of $p(\exists p \in P)$. GC_max_number is the number of the primers that is evaluated as the worst value in $P$ and $|P|$ is the number of elements in $P$. The smaller the GC_value is, the better $P$ is for GC_content.

\section{Hamming distance}

We propose this evaluation item in order to prevent mishybridization between a primer and a template or a primer and a primer.

Given two sequences $x=x_{1} x_{2} \ldots x_{l}$ and $y=y_{1} y_{2} \ldots y_{l}\left(x_{i}, y_{i} \in\{A, T, G, C\}\right)$, $H(x, y)$ indicates the hamming distance between two sequences $x, y$, which indicate the number of bases such as $x_{i}=y_{i}$. To evaluate the hamming distance between $x, y$ with different length $(|x| \leq|y|)$, we define $H_{M}(x, y)$. To count $H_{M}(x, y)$, we find total $|y|-|x|+1 H\left(x, y_{i}^{\prime}\right)$, where $y_{i}^{\prime}(1 \leq i \leq|y|-|x|+1)$ is subsequence of length $|x|$ in $y$. Additionally, in order to take into consideration the case where $x$ mishybridizes to $y$ with the sticky end at 5' or 3'end of $y$, we find total $(2|x|-2) H\left(x^{\prime}, y^{\prime \prime}\right)\left(|x|-1\right.$ at 5', 3'end, respectively), where $x^{\prime}, y^{\prime \prime}$ are the subsequences forming duplex between $x$ and $y$, respectively. $H_{M}(x, y)$ indicates the maximum in $|y|-|x|+1 H\left(x, y_{i}^{\prime}\right)$ and $2|x|-2 H\left(x^{\prime}, y^{\prime \prime}\right)$. The evaluation value (H_value) of Hamming distance on $P$ is calculated as follows. For $\exists p \in P, \exists t \in T$,

$$
H \_v a l u e=\max \left(H_{M}(p, t), H_{M}(p, \bar{t})\right)+H \_m a x \_n u m b e r / A L L \_c o m b i n a t i o n s
$$

H_max_number indicates the number of the pairs between $p$ and $t$ that is evaluated as the worst value.

$A L L_{-}$combinations indicates the number of all combinations between $p$ and $t$. 


\section{3'end_complementary}

We propose this evaluation item in order to prevent mispriming (a misextension reaction caused by mishybridization at 3 'end of a primer).

Given two sequences $x=x_{1} x_{2} \ldots x_{l}$ and $y=y_{1} y_{2} \ldots y_{l}\left(x_{i}, y_{i} \in\{A, T, G, C\}\right)$, $E(x, y)$ indicates the sum of the suffix of each base such as $x_{i}=y_{i}$ (i.e., $x=A T T G C, y=A A G G C, E(x, y)=1+4+5=10)$. To evaluate the 3'end_complementary between two sequences $x, y$ of different length $(|x| \leq|y|)$, we define $E_{M}(x, y) . E_{M}(x, y)$ indicates the maximum $E\left(x, y^{\prime}\right)$ between $x$ and $y^{\prime}(|y|-|x|+1$ subsequences of length $|x|$ in $y)$. The evaluation value (E_value) of the 3'end_complementary on $\mathrm{P}$ is calculated as follows.

For $\exists p \in P, \exists t \in T$,

$$
E_{\_} \text {value }=\max \left(E_{M}\left(p^{n}, t\right), E_{M}\left(p^{n}, \bar{t}\right)\right)+E \_m a x \_n u m b e r / A L L \_c o m b i n a t i o n s
$$

For sequence $x, x^{n}$ indicates the subsequence of length $n(0 \leq n \leq|x|)$ in $x$ from 3'end (i.e., $x=A T G T A G C C A T G G, x^{5}=C A T G G$ ).

E_max_number indicates the number of pairs between $p$ and $t$ that is evaluated as the worst value.

\section{Evaluation Function}

The evaluation value of $P$ (fitness) is calculated by the following function. The smaller fitness is, the better $P$ is.

fitness $=G C \_w e i g h t \times G C \_v a l u e^{\prime}+H \_w e i g h t \times H_{\text {_vvalue }}+E \_$weight $\times E_{\text {_value }}$

**_weight indicates the weight (positive integer) of each item.

$* * \_v a l u e '$ is translated $* *$ _value to $[0.0,1.0]$ (scale conversion).

\section{Laboratory Experiments}

\subsection{Extracting target data sequence using PCR}

Here, in order to verify that a target data sequence is extracted from NPMM by means of the above addressing method, we perform laboratory experiments for small NPMM. In the previous section, although we designed 10 primers and 27 templates, here we select 4 templates out of 27 templates and deal with a small NPMM (T4-NPMM) consisting of template to00 $_{1}$ template $_{001}$, template $_{010}$ and template $e_{011}$. These sequences are shown in Table. 1 . Thus, we can obtain data $_{000}$ by addressing $\left[B_{0}, C_{0}\right]$, data $a_{001}$ by $\left[B_{0}, C_{1}\right]$, data $a_{010}$ by $\left[B_{1}, C_{0}\right]$, and data $_{011}$ by $\left[B_{1}, C_{1}\right]$. We obtain these 4 data as described in Protocol 1 below. The results are shown in Fig. 3.

\section{Protocol 1. Amplify the target data}

Equipment and reagents

- KOD DASH DNA Polymerase (TOYOBO)

$-10 \times$ KOD DASH buffer (TOYOBO) 
Table 1. Designed sequences of templates and primers

\begin{tabular}{|c|c|c|c|c|c|}
\hline$\overline{\text { Name }}$ & Length & $\overline{\text { Sequence }}$ & $\overline{\text { Name }}$ & $\overline{\text { Length }}$ & Sequence \\
\hline$\overline{\overline{A_{0}}}$ & 15mer & GCAAAGAGCCTGTGA & $\overline{R e}$ & $15 \mathrm{mer}$ & CATCAATGTCTGGCG \\
\hline$\overline{B_{0}}$ & 15 mer & CAGTGTAAGTTCGTG & $\overline{B_{1}}$ & $15 \mathrm{mer}$ & AACGGAAAGATGCCT \\
\hline$C_{0}$ & 15 mer & TCCATGCGCTCTAAT & $C_{1}$ & $15 \mathrm{mer}$ & TACCAAACCGAGGTC \\
\hline data $a_{000}$ & 20 mer & GAGCATGTTCACTCTGGACG & data 001 & $20 \mathrm{mer}$ & AGTAAGAGTCTAGCCTAGCG \\
\hline data $a_{010}$ & $20 \mathrm{mer}$ & TTATAAACTCTCTTGACССC & $\operatorname{data}_{011}$ & $20 \mathrm{mer}$ & GCTGGCATTACACGTCTCAG \\
\hline template $_{000}$ & $80 \mathrm{mer}$ & $A_{0} B_{0} C_{0}$ data $a_{000} R e$ & template $_{001}$ & $80 \mathrm{mer}$ & $A_{0} B_{0} C_{1}$ data $_{001} R e$ \\
\hline template $_{010}$ & 80 mer & $\overline{A_{0} B_{1} C_{0} \text { data }_{010} \overline{R e}}$ & template $_{011}$ & $80 \mathrm{mer}$ & $\overline{A_{0} B_{1} C_{1} \text { data }_{011} \overline{R e}}$ \\
\hline
\end{tabular}

- dNTP MIX (2 mM of each dNTP)

- DNA thermal cycler (Biometra)

A. Primary PCR using $B_{j}(0 \leq j \leq 1)$

1. In a PCR reaction tube, add the following.

- distilled water $13.875 \mu \mathrm{l}$

- $10 \times$ PCR buffer $2.5 \mu l$

- dNTP mix $2.5 \mu l$

- T4-NPMM (each $10 \mathrm{nM}) 1 \mu \mathrm{l}$

- $B_{0}$ or $B_{1}(5 \mathrm{mM}) 2.5 \mu l$

- Re (5 mM) $2.5 \mu \mathrm{l}$

- KOD DASH Polymerase $0.125 \mu \mathrm{l}$

- Total volume is $25 \mu \mathrm{l}$

The reaction in primary $\mathrm{PCR}$ using $B_{0}$ is named $B_{0}$ after, the one using $B_{1}$ is named $B_{1 \_}$after.

2. Perform 23 cycles of PCR in automated thermal cycler:

- denature $\left(94^{\circ} \mathrm{C}\right.$ for $\left.10 \mathrm{sec}\right)$

- anneal $\left(50^{\circ} \mathrm{C}\right.$ for $\left.30 \mathrm{sec}\right)$

- polymerize $\left(72^{\circ} \mathrm{C}\right.$ for $\left.5 \mathrm{sec}\right)$

- perform the final polymerization step for an additional $60 \mathrm{sec}$

B. Secondary PCR using $C_{k}(0 \leq k \leq 1)$

1. Dilute $B_{0 \_}$after and $B_{1 \_ \text {after }} 16^{4}$ folds.

2. For each dilution, add $1 \mu l$ of each to a PCR reaction tube containing as follows:

- distilled water $13.875 \mu l$

- $10 \times$ PCR buffer $2.5 \mu l$

- dNTP mix $2.5 \mu l$

- $C_{0}$ or $C_{1}(5 \mathrm{mM}) 2.5 \mu \mathrm{l}$

- Re (5 mM) $2.5 \mu l$

- KOD DASH Polymerase $0.125 \mu \mathrm{l}$

- Total volume is $25 \mu \mathrm{l}$

The reaction in $B_{0 \_}$after using $C_{0}$ is named $B_{0} C_{0 \_}$after, the one using $C_{1}$ is named $B_{0} C_{1 \_}$after; the reaction in $B_{1 \_}$after using $C_{0}$ is named $B_{1} C_{0 \_}$after, the one using $C_{1}$ is named $B_{1} C_{1 \_ \text {after. }}$.

3. Perform 23 cycles of PCR using the same cycle parameters as for the primary PCR (part A, step 2)

4. Run $5 \mu \mathrm{l}$ of the 6 reactions on a $10 \%$ PolyAcrylamide Gel, then visualize by ethidium bromide. Determine whether a strong band of the expected size is obtained for each reaction (Fig. 3). 


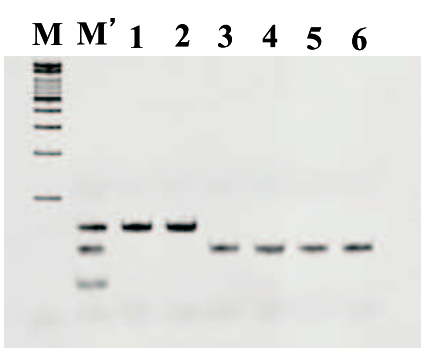

Fig. 3. M: 100 bp ladder. M': DNA marker of length 65 , 50, and 35 bp. Lane 1: B0_after. Lane 2: B1_after. Lane 3: $B_{0} C_{0 \_}$after. Lane 4: $B_{0} C_{1} \_$after. Lane 5: $B_{1} C_{0} \_$after. Lane 6: $B_{1} C_{1} \_$after.

\subsection{Detection of amplified sequence}

In Fig. 3, we can expect that each lane (numbered 3-6) consists of only one sequence coding: data $a_{000}$, data $a_{001}$, data $a_{010}$ and $d a t a_{011}$, respectively. To confirm this, we make the following experiments with PCR, because $50 \mathrm{bp}$ is not an adequate length to read out the sequence by a sequencer.

For diluted $B_{0} C_{0 \_} a f t e r$, we perform PCR using Re and data $a_{000}$ primer, which is the sequence of length 15 in data $a_{000}$ at 5 'end (shown in Table. 2). In the same way, PCR is performed on the diluted $B_{0} C_{0 \_}$after with data $a_{001}$ primer, data $_{010}$ primer, data ${ }_{011}$ primer, and $R e$. If, and only if, a sequence of length 35 bp is amplified by using data $a_{000}$ primer, then $B_{0} C_{0 \_}$after consist of the only sequence containing $d a t a_{000}$. Thus to check out the sequence in $B_{0} C_{0} \_$after, a total of $4 \mathrm{PCR}$ is needed. For $B_{0} C_{1 \_}$after, $B_{1} C_{0 \_}$after, and $B_{1} C_{1 \_}$after, we performed the same operations described in Protocol 2 below.

Table 2. Sequence of data primers

\begin{tabular}{||c|c|c||c|c|c||}
\hline \hline Name & Length & Sequence & Name & Length & Sequence \\
\hline \hline${\text { data } a_{000} \text { primer }}$ & $15 \mathrm{mer}$ & GAGCATGTTCACTCT & data $a_{001}$ primer & 15mer & AGTAAGAGTCTAGCC \\
\hline data $a_{010}$ primer & $15 \mathrm{mer}$ & TTATAAACTCTCTTG & dat $_{011}$ primer & 15mer & GCTGGCATTACACGT \\
\hline \hline
\end{tabular}

\section{Protocol 2. Detection by using data primers}

1. Dilute $B_{0} C_{0 \_}$after, $B_{0} C_{1} \_$after, $B_{1} C_{0 \_}$after and $B_{1} C_{1 \_}$after $16^{4}$ folds.

2. Add $1 \mu l$ of each dilution to a PCR reaction tube containing the following (for each diluted mix, each data primer is used, therefore a total of 16 reactions are needed).

- distilled water $13.875 \mu l$

- $10 \times$ PCR buffer $2.5 \mu l$

- dNTP mix $2.5 \mu l$

- data $a_{000}$ primer, data $a_{001}$ primer, data $a_{010}$ primer, or data $a_{011}$ primer $(5 \mathrm{mM}) 2.5 \mu \mathrm{l}$ 
- $\operatorname{Re}(5 \mathrm{mM}) 2.5 \mu \mathrm{l}$

- KOD DASH Polymerase $0.125 \mu l$

- Total volume is $25 \mu l$

3. Perform 25 cycles of PCR using the same cycle parameters as for the primary PCR (part A, step 2 in Protocol 1).

4. Run $5 \mu l$ of each of the 16 reactions on a $10 \%$ PolyAcrylamide Gel, then visualize by ethidium bromide (shown in Fig. 4).

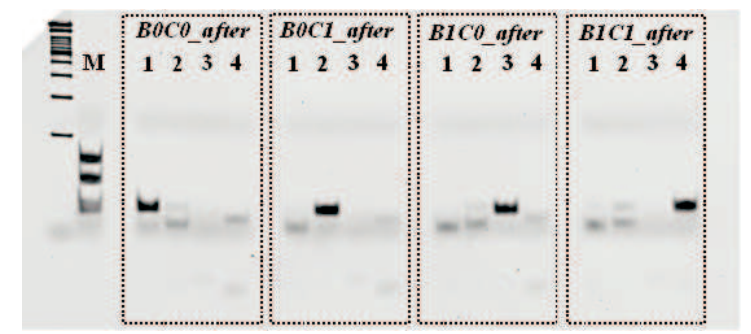

Fig. 4. M: DNA marker of length 65,50 , and $35 \mathrm{bp}$. The index of 1: use data $a_{000}$ primer. 2: use data $a_{001}$ primer. 3: use data $a_{010}$ primer. 4: use data $a_{011}$ primer

\section{Discussions}

Figure 4 indicates that almost the only sequence containing data $_{000}$ exists in $B_{0} C_{0}$ _after. The desired results are obtained for other solutions as well. Although some weak bands appear, those are primer dimmers because weak bands also appear in spite of amplifying only one template with data primers (data not shown). From these results, we can conclude that a desired target data is extracted from T4-NPMM.

\subsection{Amplification using concatenation primer}

Here, we perform PCR for T4-NPMM with a concatenation primer made up of two consecutive primers (in this experiment, we use $B_{0} C_{0}$, which is $B_{0}$ concatenated $C_{0}$ ). The purpose of this experiment is to see whether we can extract the target data by only one PCR procedure. Of course, whether that is possible depends on annealing temperature $\left(T_{a}\right)$. We perform the experiment as described in Protocol 3 below. We can regard this PCR as competitive PCR between template $_{000}$ and template te10 , so the number of cycles in PCR using $B_{0} C_{0}$ is not a critical parameter [10].

Protocol 3. Amplify the target data using $B_{0} C_{0}$

A. PCR to T4-NPMM using $B_{0} C_{0}$

1. in a PCR reaction tube, add the following.

- distilled water $13.875 \mu \mathrm{l}$

- $10 \times$ PCR buffer $2.5 \mu l$ 


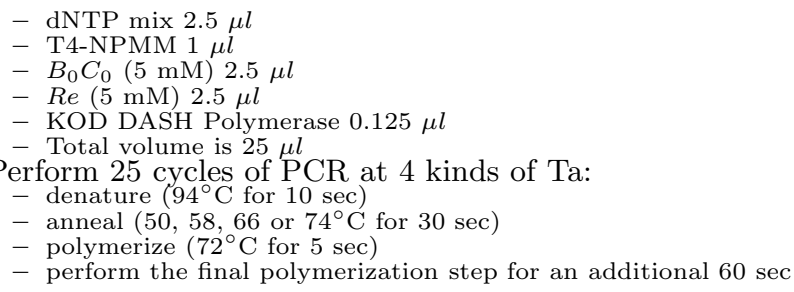

B. Detection of sequence amplified with $B_{0} C_{0}$

1. Dilute the reactions at each annealing temperature $16^{4}$ folds.

2. Add $1 \mu l$ of each dilution to a PCR reaction tube as in Protocol 2: - distilled water $13.875 \mu \mathrm{l}$

$-10 \times$ PCR buffer $2.5 \mu l$

- dNTP mix $2.5 \mu l$

- data 000 primer, data 001 primer, data $a_{010}$ primer, or data ${ }_{011}$ primer $(5 \mathrm{mM}) 2.5 \mu \mathrm{l}$

- Re (5 mM) $2.5 \mu \mathrm{l}$

- KOD DASH Polymerase $0.125 \mu l$

- Total volume is $25 \mu l$

3. Perform PCR using the same cycle parameters as in Protocol 1, and set aside each solution from the thermal cycler every 2 cycles from 17 to 25 cycles.

4. Run $5 \mu \mathrm{l}$ of all the reactions (80 samples) on a $10 \%$ PolyAcrylamide Gel, then visualize by ethidium bromide.

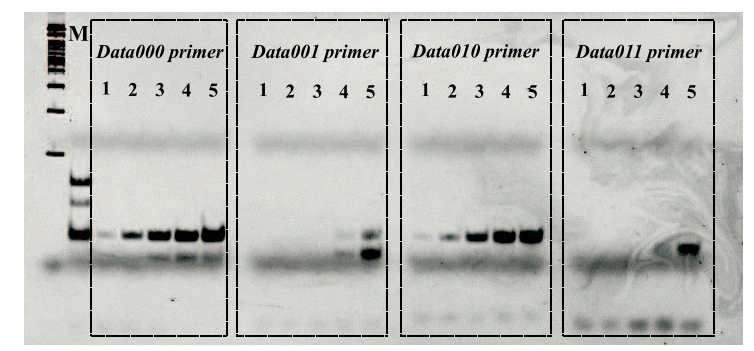

Fig. 5. Results of Detection Experiment, as in the previous subsection for T4-NPMM amplified by using $B_{0} C_{0}$ at Ta $66^{\circ} \mathrm{C}$. M: DNA marker of length 65,50 , and $35 \mathrm{bp}$. Index of 1: the solution set aside from the thermal cycler at the 17 th cycle. $2: 19$ th cycle. 3 : 21 st cycle. 4 : 23 rd cycle. 5: 25 th cycle.

\section{Discussion}

In the case of PCR using $B_{0} C_{0}$ at Ta 50,58 , or $66^{\circ} \mathrm{C}$, some sequences are amplified. In the other case at annealing temperature $74^{\circ} \mathrm{C}$, no sequence is amplified. Figure 5 shows the results of the data detection experiment after the PCR solution at $66^{\circ} \mathrm{C}$. From the fact that the solutions amplified with data $a_{000}$ primer and dat $_{010}$ primer are visualized at almost the same step rate, the solution contains two sequences including $d a t a_{000}$ and $d a t a_{010}$, respectively. This indicates that mispriming occurs between template te10 $_{0}$ and $B_{0} C_{0}$ in spite of a high temperature such as $66^{\circ} \mathrm{C}$. Therefore, we believe that decreasing the times of PCR would be difficult. (We have no data at higher $T_{a}$ than $66^{\circ} \mathrm{C}$ (below $74^{\circ} \mathrm{C}$ ), but this is no concern if each primer is lengthened.) 


\section{Concluding Remarks and Future Works}

In this paper, we proposed a DNA memory with high capacity, high data security and high specificity of chemical reaction and we showed the feasibility of NPMM through some experiments.

In the future, we should consider the design strategy for a set of primers that can be used without dependence on the data sequence. Also, we should investigate the scaling-up of NPMM. Since NPMM has the potential for high reaction specificity, even if the size of NPMM became larger, we believe that NPMM would work appropriately. Scaled-up NPMM will be realized in the near future.

\section{References}

1. T. Head and M. Yamamura, S. Gal: "Aqueous Computing. writing on molecules", Proceedings of CEC 99, (Congress on Evolutionary Computation), pp. 1006-1010 (1999)

2. T. Head, G. Rozenberg, R. S. Bradergroen, C. K. D. Breek, P. H. M. Lommerse and H. P. Spaink: "Computing with DNA by operating on plasmids", Biosystems, Vol.57, pp. 875-882 (2000)

3. E. B. Baum: "Building an Associative Memory Vastly Larger Than the Brain", Science, Vol.268, pp. 583-585 (1995)

4. J. H. Reif and Thomas H. LaBean: "Computationally Inspired Biotechnologies: Improved DNA Synthesis and Associative Search Using Error-Correcting Codes and Vector-Quantization", Sixth International Meeting on DNA Based Computers (DNA6), in Lecture Notes in Computer Science, pp. 145-172 (2001)

5. J. H. Reif, Thomas H. LaBean and Michael Pirrung: "Experimental Construction of Very Large Scale DNA Databases with Associative Search Capability", Seventh International Meeting on DNA Based Computers (DNA7), in Lecture Notes in Computer Science, pp. 231-247 (2002)

6. M. Arita, A. Nishikawa, M. Hagiya, K. Komiya and H. Gouzu and K. Sakamoto: "Improving Sequence Design for DNA Computing", Proceedings of GECCO'00 (Genetic and Evolutionary Computation Conference), pp. 875-882 (2000)

7. M. Garzon, R. Deaton, L. F. Nino and Ed Stevens: "Encoding Genomes for DNA Computing", Proceedings of the Third Annual Genetic Programming Conference, pp. 684-690 (1998)

8. F. Tanaka, M. Nakatsugawa, M. Yamamoto, T. Shiba and A. Ohuchi: "Developing Support System for Sequence Design in DNA Computing", Seventh International Meeting on DNA Based Computers (DNA7), in Lecture Notes in Computer Science, pp. 129-137 (2002)

9. M. J. McPherson, P. Quirke and G. R. Taylor: "PCR A Practical Approach", IRL PRESS, (1991)

10. M. J. McPherson, B. D. Hames and G. R. Taylor: "PCR2 A Practical Approach", IRL PRESS, (1995). 